\title{
Highlights from the Multi Unit Spectroscopic Explorer (MUSE): a 2nd generation VLT instrument for the VLT (Presentation Video)
}

\section{Roland Bacon}

Roland Bacon, "Highlights from the Multi Unit Spectroscopic Explorer (MUSE): a 2nd generation VLT instrument for the VLT (Presentation Video)," Proc. SPIE 9147, Ground-based and Airborne Instrumentation for Astronomy V, 914750 (22 June 2014); doi: 10.1117/12.2063673

Event: SPIE Astronomical Telescopes + Instrumentation, 2014, Montréal, Quebec, Canada 


\title{
Highlights from the Multi Unit Spectroscopic Explorer (MUSE): A 2nd generation VLT instrument for the VLT (Presentation Video)
}

\author{
Roland Bacon, Observatoire de Lyon (France) and MUSE Consortium
}

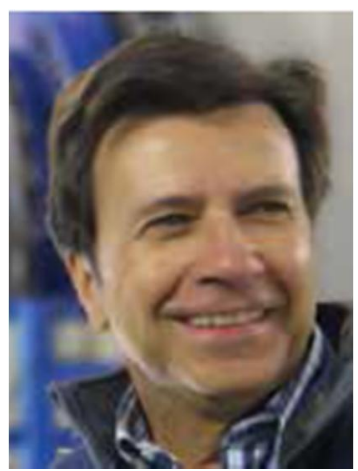

\begin{abstract}
The Multi Unit Spectroscopic Explorer (MUSE) is a second-generation VLT panoramic integral-field spectrograph. The instrument has been designed to take advantage of the VLT ground layer adaptive optics ESO facility using four laser guide stars. MUSE couples the discovery potential of a large imaging device to the measuring capabilities of a highquality spectrograph, while taking advantage of the increased spatial resolution provided by adaptive optics. The MUSE hardware is composed of 24 identical modules, each one consisting of an advanced slicer, a spectrograph and a (4k) ${ }^{2}$ detector. A series of fore-optics and splitting and relay optics is in charge of derotating and partitioning the square field of view into 24 sub-fields. With its almost 7 tons of opto-mechanics, MUSE is one of the biggest integral field unit ever built. After a successful preliminary acceptance in Europe in fall 2013, MUSE has been dismounted, shipped to Chile and re-integrated in the Paranal new integration hall and finally installed on the Nasmyth platform of UT4 late January this year. During the 2 commissioning runs, hundreds of millions of spectra have been obtained in order to validate the instrument and measured its achieved performance. To demonstrate its power, a number of show-case and spectacular observations have also been obtained. Preliminary results demonstrate that MUSE is likely to become a new reference in the field of integral field spectroscopy thanks to its large field of view, very high throughput, excellent image quality, good spectral resolution, wide simultaneous spectral range and state-of-the art control and data reduction software. I will review this success story, from the call of idea to the deployment on the VLT, including the latest performances and showcase observations.
\end{abstract}

View presentation video on SPIE's Digital Library: http://dx.doi.org/10.1117/12.2063673.3664789952001 